Article

\title{
\#ThisIsMeChallenge and Music for Empowerment of Marginalized Groups on TikTok
}

\author{
Arantxa Vizcaíno-Verdú * and Ignacio Aguaded \\ Department of Pedagogy, University of Huelva, Spain \\ * Corresponding author (arantxa.vizcaino@dedu.uhu.es)
}

Submitted: 19 July 2021 | Accepted: 21 September 2021 | Published: 24 February 2022

\begin{abstract}
Media convergence is generating many collective performances on social media, where the rise of short-form videos has created a new opportunity for the empowerment of society on online platforms. In this context, TikTok appears as an application for creative expression through music clips and lip-syncs. Through the \#ThislsMeChallenge hashtag, which introduces the musical theme of The Greatest Showman film, we analyze a new online practice within messages from traditionally marginalized groups throughout individual and collective life events. In order to understand TikTok as a music venue for social empowerment, we conducted a quantitative content analysis of 100 TikTok posts under the hashtag, and an artificial intelligence sentiment analysis across 8,877 comments. The results show a wide range of performance work that addresses issues of gender, sexual orientation, racial discrimination, and other types of current hate speech. In short, we conclude that TikTok has become a platform that seems to motivate activism and empowerment of marginalized groups through music frameworks that challenge social discrimination.
\end{abstract}

\section{Keywords}

\#ThisIsMeChallenge; empowerment; marginalized groups; social media; TikTok; transmedia music; video activism

\section{Issue}

This article is part of the issue "New Narratives for New Consumers: Influencers and the Millennial and Centennial Generations" edited by Luis M. Romero-Rodríguez (Rey Juan Carlos University), Santiago Tejedor (Autonomous University of Barcelona), and Inmaculada Berlanga (International University of La Rioja).

(C) 2022 by the author(s); licensee Cogitatio (Lisbon, Portugal). This article is licensed under a Creative Commons Attribution 4.0 International License (CC BY).

\section{Introduction}

Social media has become an essential space for the study of empowerment in terms of identity negotiation among individuals, and as a window into the self-expression by users interacting with each other and for each other (Literat \& Kligler-Vilenchik, 2021). In this "post-Internet" environment merging digital networks, portable devices, and peer-to-peer knowledge sharing, users experience positive feedback which reflects the community's possibilities for motivation and perceived belongingness (Waugh, 2017). In other words, participatory culture facilitates individuals interacting in different digital networks to represent their identities through social perceptions and expectations (Literat et al., 2021).
TikTok plays a significant role in this context. The platform, available internationally in Apple, Android, and Amazon app stores since 2018, is an algorithm-driven app that presents short video content to users via various interaction and navigation options-likes, follows, views, among other affordances. The app is characterized by a video feed, "For You Page," in which users view one video at a time, swiping up or down for new or previous posts, and by an extensive music and audio library that supports each one. A spinning circle appears at the bottom of each video to click and learn more about the audio: videos that have used it, the song itself, or the option to add it to your favorites (Anderson, 2020). This sound appropriation process has generated a wide range of popular content and challenges, as well as a wave 
of audio and video memes-audios and videos that are continuously replicated, acquiring and adding meanings of belonging among the user community (Zulli \& Zulli, $2020)$. As noted by journalists and communication scholars Fang et al. (2019) in their study on the concept of "anesthesia" in short video apps, this dynamic consumption of content evolves into an "anesthetic effect" that provokes users to consume TikTok posts for long periods of time out of curiosity, similar to the algorithmic system of YouTube.

What makes TikTok particularly interesting is that as indicated by Internet anthropologist Abidin (2021) in her platform mapping from the perspective of celebrities, communities, and topic thresholds in this app have led to controversy and content removal when TikTokers-users who create content on the app-reach their popularity due to issues of social (in)justice. Since its launch, this social networking site has been trying to reach young people through silly, shameless, and unfiltered content. As a result, the app avoids the seriousness and the perfect variety of filters of Instagram, the ephemeral stress of Snapchat's 24-hour content, or the verbal warfare of Twitter's discussion threads (Kennedy, 2020). TikTok prevents these formats inviting users to create videos under the guise of normality, creativity, and realness, concealing the toxic nature of harassment and other social media conflicts. In other words, masking the "aggressive architecture" of the Internet (Harvey, 2019), which is based on urban studies, through which governments discourage some kinds of activities in public spaces. Such "aggressive architecture" on the Internet refers to hostile innovations that prevent undesirable interactions on platforms, constricting what creators consider desirable publics, and systematically disadvantaging those who have not been valued in the digital environment. Considering this, digital content created in other platforms around different minority communities such as immigrants (Civila et al., 2020; Jaramillo-Dent et al., 2021), LGBTIQ+ (Craig et al., 2021), people with disabilities (Harb \& Sidani, 2020), among others, have resulted in representations involving expectations, prejudices, or prevailing norms. Albeit, individuals are not handicapped by these social and digital perspectives, cultivating close relationships with others that share similar interests to offer a more honest portrayal of themselves (Sachs et al., 2021). This insight is grounded in the online hate moods analyzed by media and culture scholar Kilvington (2021), who introduces a hateful belief that represses users on the Internet. Hence, content sharing on social media such as TikTok emerges as a liberating process for individuals and communities to express themselves, providing a diversity of identities that pursue positive emotions (Wahl-Jorgensen, 2020).

In this regard, music has become a source to share life experiences (Born \& Tilley, 2011), which is the main feature of TikTok. The opportunities for identity self-expression lead to new forms of digital culture on TikTok, involving a music-making relationship that is understood as a democratization of music-based content (Chambers, 2021). Such approach is further strengthened on a study conducted by psychologists and musicians Anderson et al. (2020) about musical self-representation on Spotify, which concludes that personality is conveyed through musical preferences and performances. In this case, we discuss appropriation and musical sharing on TikTok through hashtags and music challenges, comprised as a trend preceded by hashtag symbol "\#" to promote online events, discussions, and social phenomena (Vizcaíno-Verdú \& Abidin, 2021). Thus, we are faced with social trends of self-representation and community imaginaries based on transmedia music, which is a practice for content spreading through userappropriated music aimed at disseminating new storytelling meanings (Vizcaíno-Verdú et al., 2021).

Focused on the self-expression of identity through this short video storytelling perspective, we propose a study that aims to explore the role of the music \#ThislsMeChallenge phenomenon on TikTok to connect traditionally marginalized groups within an empowering digital activism posts. This music challenge comprises the hashtag and audio from "This Is Me" by Michael Gracey's musical drama film "The Greatest Showman," addressing the social demotion of "freak performers" in "Barnum's Circus." The challenge consisted of presenting discrimination and coping experiences by marginalized groups, seeking to emphasize their identity and value as a symbol of empowerment.

\section{Social Media Movements and Empowerment}

The plethora of social media has created a set of collective and multidimensional interactions characterized by constantly varying causes of social justice. From a sociological-anthropological approach, we need to understand a social movement as a collective, voluntary, and coordinated action, articulated around a cause or claim that defines an opponent or situation to be changed (Asún \& Zúñiga, 2013). This means that social media movements pursue collaboration between individuals who combine efforts to express themselves as a group, trying to change or improve a situation of disadvantage (García-Galera et al., 2017). Beyond this macro-concept, we focused on the empowerment phenomenon. Empowerment is understood as the result of a process-social movement-which may occur in different degrees and areas of life, where power relations between individuals and social groups undergo transformation through three critical processes: (a) changing ideologies that justify inequalities; (b) changing patterns of economic, natural or intellectual access, or control; (c) changing structures that reinforce and maintain unequal powers; and (d) changing prejudices and preconceived values that denigrate people's human capabilities and conditions (Maiorano et al., 2021). This networkbased power of communication and content convergence serves as an alternative tool for the discussion and 
expression of communities that claim their rights and motivate equality (Kumari, 2020).

\subsection{Discrimination and Marginalized Groups on the Internet}

Collectives and individuals who are marginalized through discriminatory conditions such as racism, homophobia, ableism, gender identity, religion discrimination, ideology, among others (Place, 2021), employ emancipatory technologies such as digital platforms to expose the truth and drive social change (George et al., 2021). In this sense, in this study we considered significant understanding social discrimination and marginalized groups, since the song analyzed was related to coping the social exclusion.

From the perspective of social behavior, discrimination refers to the unequal treatment of individuals or groups based on racial, religious, physical, political, gender, age, sexual orientation, among other aspects (Velasquez \& Montgomery, 2020). At this point, social media has caused great discrimination towards marginalized groups, which are communities excluded from mainstream society, as well as from economic, educational, and cultural life (Parson, 2019). This leads people to face prejudice, bullying, exclusion, violence, in the hopes for equality and social acceptance (Meyer, 2019). On the internet, intergroup contacts made these marginalized groups more sensitive to possible identity prejudice (Mancini \& Imperato, 2020) and collective activism (Smith et al., 2020). In this regard, human behavior scholars Spears and Postmes (2015), on a study focused on group identity and social influence, pointed out that this online activist motivation can be turned into presentations of the self that reinforce in-group and outgroup social identities.

\subsection{Sympathy, Empathy, Representation, and Reflexivity on TikTok}

The social media movements could be observed on platforms such as TikTok, where video activism - understood as an audio-visual complaint located outside governmental and marketing logics-is increasing (Askanius, 2013). This sort of message can attract the sympathy of public opinion and transform it into empathy (Chanan, 2011). This means that sympathy, which is understood as a subjective action, generally aimed at rescue, aid, or assistance (Olinick, 2014), may be transformed into an active and empathetic community. Thus, we understand empathy as the capacity to relate to another's emotional state, grounded in the emotion regulation theory-which is based on sharing sympathetic and familiar expressions (Yalcin \& DiPaola, 2018). In this regard, video activism is transferred to TikTok and relies on the appropriation of a media and storytelling discourse through representation (the set of words, statements, and emotions concerning an injustice or inequality) and reflexivity (the set of confronted values where community notions are expressed; Meneses-Rocha \& Castillo-González, 2016). Therefore, reflexivity is a resilient process by which people express values that enhance a community sense of freedom, choice, and empowerment (Givropoulou \& Tseliou, 2021).

On TikTok, we may find two different scientific perspectives. On the one hand, media scholars Weimann and Masri (2020) point out that the innocence of the platform is pushed back to a sinister side: the hate speech phenomenon. On the other hand, theologian Zhang (2020), in his study on the "infrastructuralization" of the platform as an entertainment channel, argues for an app that deepens power relations between the different groups of users. For this reason, in this article we try to understand the second perspective through the potential of TikTok music challenges for empowering different grouping social movements.

\section{Methodology}

We conducted a qualitative walkthrough method, and a quantitative and correlational analysis using content analysis and natural language processing with sentiment analysis (SA). The walkthrough method led us to understand the music challenge phenomenon by immersing ourselves daily on TikTok (Light et al., 2016). Quantitative content analysis is a systematic method concerning the characteristics of messages through accepted meanings (Neuendorf, 2016). These approaches allowed us to examine the social movements that characterize \#ThislsMeChallenge posts on TikTok. On the other hand, SA enabled the computational exploration of polarized and subjective opinions expressed by users viewing these contents (Medhat et al., 2014). Thus, these methods provided an understanding of both TikToker content (creator of content) and the user (receiver of content). We defined the following research questions to carry out the analysis:

RQ1: What role does music play on TikTok's \#ThisIsMeChallenge posts?

RQ2: Is there a correlation between the representation and reflexivity (empowerment) of TikTok groups participating in the \#ThislsMeChallenge?

RQ3: The comments of \#ThisIsMeChallenge posts on TikTok support the appearing marginalized groups?

\subsection{The Hashtag Case \#ThislsMeChallenge}

For the analysis of empowering social movements described by Place (2021) on TikTok, we selected the hashtag \#ThisIsMeChallenge. This challenge, which had received $71.4 \mathrm{M}$ views at the time of the analysis in February 2021, introduces a clip of the song "This Is Me" from the film The Greatest Showman released in 
October 2017. This pop song was the promotional single for the film and won a Golden Globe for Best Original Song. We chose this challenge because of its popularity in terms of views, and the emotional impact on the individuals who participated in it.

\subsection{Walkthrough Method and Quantitative Content Analysis of Video Empowerment}

The first stage to understand the empowerment phenomenon on TikTok through the hashtag \#ThisIsMeChallenge was use of music for storytelling on posts. We intended to comprehend how music moves beyond the fictional environment through other meanings (transmedia music; Vizcaíno-Verdú et al., 2021). To do so, we performed a walkthrough method (Light et al., 2016):

1. Registration and entry: Creation of a unique TikTok profile for analysis.

2. Everyday use: Immersion for one hour daily from February 7-18, identifying how TikTok music (audio) relates to visual content (image).

3. App suspension, closure, and leaving: Ending the analysis and interpreting the observations.

Content analysis is an empirical-systematic method used to analyze audio, text, and image data (Krippendorff, 2004). This process consists of analyzing content variables through units of analysis and a codebook. The units of analysis are the measurable content, and the codebook is a collection of observable codes (Bock et al., 2011). In the quantitative method process, these codes are labeled using a numerical system that facilitates the statistical analysis.

In this study we built a codebook based on studies about digital empowerment to define the sociodemographic variables of TikTokers (George et al., 2021), on the representation and reflexivity of identity (MenesesRocha \& Castillo-González, 2016), as well as on hate speech on TikTok (Weimann \& Masri, 2020) to analyze the performances (see complete codebook at https:// doi.org/10.6084/m9.figshare.14903064.v1). As we highlighted above, representation referred to unjust or unequal issues (Meneses-Rocha \& Castillo-González, 2016), and reflexivity concerned a resilience process whereby a community expresses its value (Givropoulou \& Tseliou, 2021).

We validated this codebook using the Fleiss' kappa statistical test in the SPSS v. 25 software. The procedure consisted in testing the inter-rater agreement on the "representation" and "reflexivity" variables. The test was selected based on 15 random videos from the sample detailed below, involving the same rater in two different periods-December 2020 and March 2021 (Fleiss et al., 2003). For the variable "representation" we obtained a Fleiss' kappa coefficient $k=.751, p=.001$, which reflected a statistically significant good agreement, according to Cohen's kappa classification (between $.61-.80$ is good). With regards to the variable "reflexivity" we scored a Fleiss' kappa coefficient $k=.825$, $p=.001$, which also represented a statistically significant very good agreement (between $.81-1.00$ is very good). Then, we proceeded to the descriptive and correlation analysis.

\subsubsection{First Sample: The Creators}

The sample comprising the first analysis was manually collected through the TikTok platform by following the next sequence:

1. Searching the \#ThisIsMeChallenge hashtag on the "Discover tab";

2. Filtering the results by the "videos tab";

3. Filtering the contents by "All time" in "Date posted" and sorting by "Relevance."

The videos were registered and stored considering the following selection criteria: (a) posts featuring only one person and (b) posts employing the sounds "Original sound-itsbybrandon," "Original sound-Maria Clarin," "This Is Me-Keala Settle \& The Greatest Showman Ensemble," "This Is Me (From The Greatest Showman)-Kesha," which introduced similar musical versions. The dataset consisted of 100 videos released between 2019 and 2021. The sample was targeted to answer the first two research questions, related to the TikToker (creator) and social movements.

\subsection{SA of User Comments}

SA is a natural language processing task aimed at mining opinions from texts. This technique is mainly used in the fields of affective computing research and emotion recognition (Poria et al., 2017), which are key to the development of artificial intelligence. This system is considered a text classification task because it performs two analyses: polarity (the level of positivity or negativity of a text) and subjectivity (the level of subjectivity or objectivity of a text; Birjali et al., 2021).

For the analysis of polarity and subjectivity we used the TextBlob library through a line of code with Python programming language in PyCharm. PyCharm is an integrated development environment for computer programming. Through this system we collected the sample detailed below and checked whether user comments viewing the 100 TikTok posts were mainly positive and subjective, i.e., whether they turn the viewers' sympathy into empathy. We related the polarity-subjectivity variables to sympathy-empathy by looking at the comments, considering those that introduced sympathetic expressions according to the emotional regulation theory (Yalcin \& DiPaola, 2018). 


\subsubsection{Second Sample: The Users}

The second sample, related to the third research question, comprised comments from users. We decided to apply the TextBlob library tool to comments because it worked exclusively with text, preventing the reading of files such as images or videos. Also, we decided to analyze the comments that users made on the videos to get an insight into how they felt about the marginalized groups in the \#ThislsMeChallenge.

To maintain an equal data pattern, we first downloaded 150 comments for each video. The data was then comprised in a dataset of 15,000 comments and transformed into an Excel spreadsheet. Then we processed the text on SA and excluded from the spreadsheet comments that obtained neutral values in polarity and subjectivity. When filtering the data on Excel, we realized that the detection system did not recognize text with emoji, words with split letters (e.g., "w o n d e r f u l"), and tags (e.g., @userexample). In other words, all the comments that did not give information were removed. Once filtered, we analyzed 8,877 comments through an automated content analysis which consisted of measuring the mean polarity-subjectivity of the entire sample. We also conducted a qualitative content analysis to group the comments by behavioral categories that would allow us to understand the community's perspective on marginalized groups.

Before submitting the data to the SA, we translated the comments into English using the TextBlob library. We identified comments in English, Spanish, French, German, Japanese, Korean, Thai, Indonesian, Malay, Danish, Tagalog, Russian, Swedish, Croatian, Italian, Catalan, Portuguese, Vietnamese, Arabic, Finnish, Dutch, Greek, Polish, and Norwegian.

\subsection{Illustrated Examples and Ethical Concerns}

The TikTok posts presented in this article were included after checking that their creators intended to circulate their posts publicly on the platform. Such reasoning was supported as the sample of 100 selected videos was derived from the "Relevance" tab of TikTok, which refers to the most searchable and findable posts by users. Thus, the posts illustrated below are considered as "superpublic" content (boyd, 2013), which consists of data that pursues high visibility (Abidin, 2016). In addition, to avoid infringing the image rights of individuals, the authors created illustrations about the most characteristic examples, anonymizing the data profiles.

\section{Results and Discussion}

\subsection{The Music Role on the \#ThislsMeChallenge Phenomenon}

Following the walkthrough method, we delve into the music's role in the challenge. As we previously com- mented, this hashtag refers to the song "This Is $\mathrm{Me}^{\text {" }}$ composed by Keala Settle and released as a single in The Greatest Showman: Original Motion Picture Soundtrack for the 2017 film The Greatest Showman. During the analysis we found the following audios: "Original sound-itsbybrandon," "Original sound-Maria Clarin," "This Is Me-Keale Settle \& The Greatest Showman Ensemble," "This Is Me (From the Greatest Showman)-Kesha," and "Original sound-Linda Marin Sanchez." Nevertheless, we found that the most significant sections of the song were repeated to highlight individual stories. According to the musical translation project of The Greatest Showman soundtrack (García-Moreno, 2020), we focused on organizing the challenge in two forms: audio-meaningful storytelling and image.

Firstly, we observed the same pattern in all the audios, i.e., mixing chorus sections in a different way than in the original song. As shown in Figure 1, the videos always started with "I am brave, I am bruised," referring to the TikTokers self-presentation. This kind of introduction could be related to the argument that marginalized groups are usually motivated to reinforce their in-group and out-group social identities on social media (Spears \& Postmes, 2015).

During the next musical transition starting with the lyrics "Look out "cause here I come" (1st, 2nd, and 3rd sections), the TikTokers linked several transitions gradually making-up their faces. Throughout this sequence, they kept a peaceful facial expression ending as the song claimed not to apologize for being who they are. Then, a new video transition showed the lyrics "When the sharpest words wanna cut me down" (4th section) with the female vocalist, without musical instruments, in which the TikTokers showed insulting words over their unhappy face. The most frequent insults were collected in Figure 2.

Once the sad section ended with "This Is Me" lyrics, we listened to the final chorus where all the music instruments were played again (5th section). Just like in the film, in this final video sequence we found several transitions, colors, and the challenging happiness of the TikTokers, showing that they successfully coped with all their social discrimination issues. Thus, we visually identified the following challenge stages (Figure 3).

\subsection{Representation and Reflexivity Through \#This/sMeChallenge}

To answer the second research question, related to the 100 posts of the challenge, we performed a correlational statistical analysis between the code "representation" and "reflexivity." The aim was finding out whether there was any relationship between both variables involved in identifying the injustice perceived by the individual (referring to a marginalized group or social movement) and how this was a symbol of reflexivity throughout resilient stories (empowerment). 




Figure 1. Comparing the original "This Is Me" lyrics and the audio's TikTok lyrics.

Considering that our aim in this stage of the analysis was to relate both variables, prior to the selection of the correlation statistical test we administered the Kolmogorov-Smirnov test of the equality of continuous and one-dimensional probability distributions (as $n \geq 50$ ). This test confirmed that the significance was not higher than $K-S(100)=.36, p=.001$. Following this criterion, we then followed the Spearman's test, which is a nonpara- metric measure of the strength and direction of association between two variables.

After mining the data, we found that some of the "representation" variables did not occur within the sample (for example, injustices or inequalities caused by age, language, ideology, or poverty). Below, we analyze the identified variables. In this sense, we coded the following number of videos for each one: racism

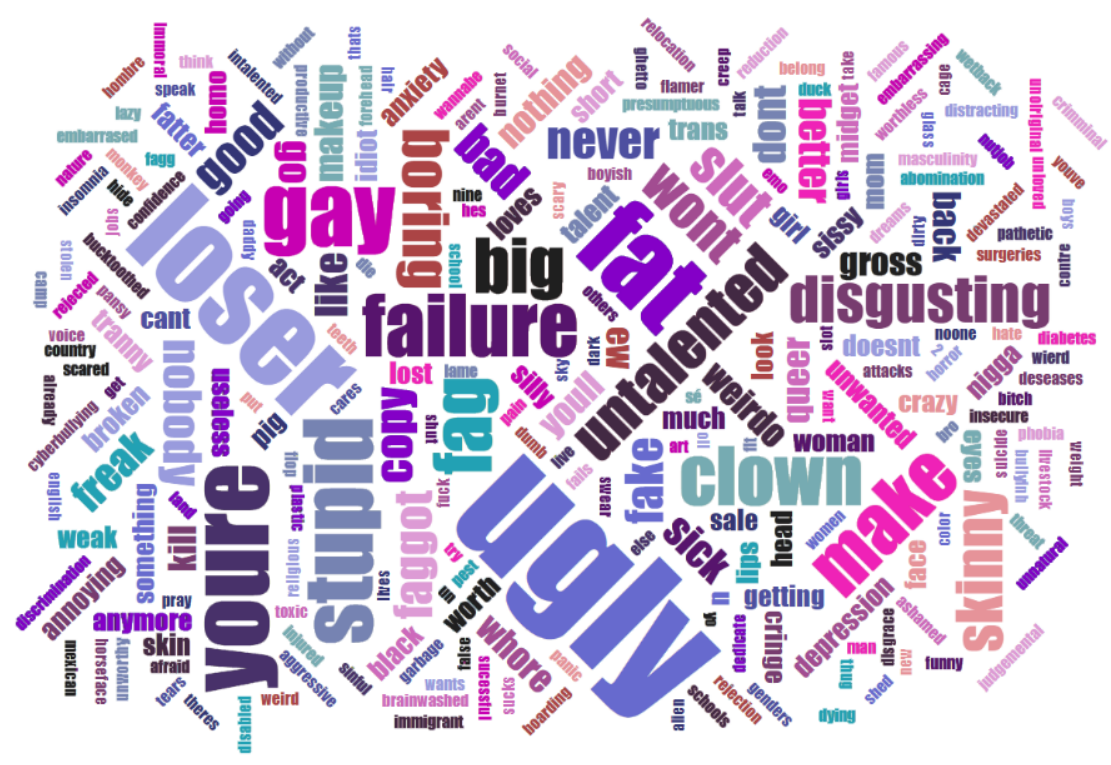

Figure 2. Self-perceived insults by TikTokers in \#ThisIsMeChallenge. 

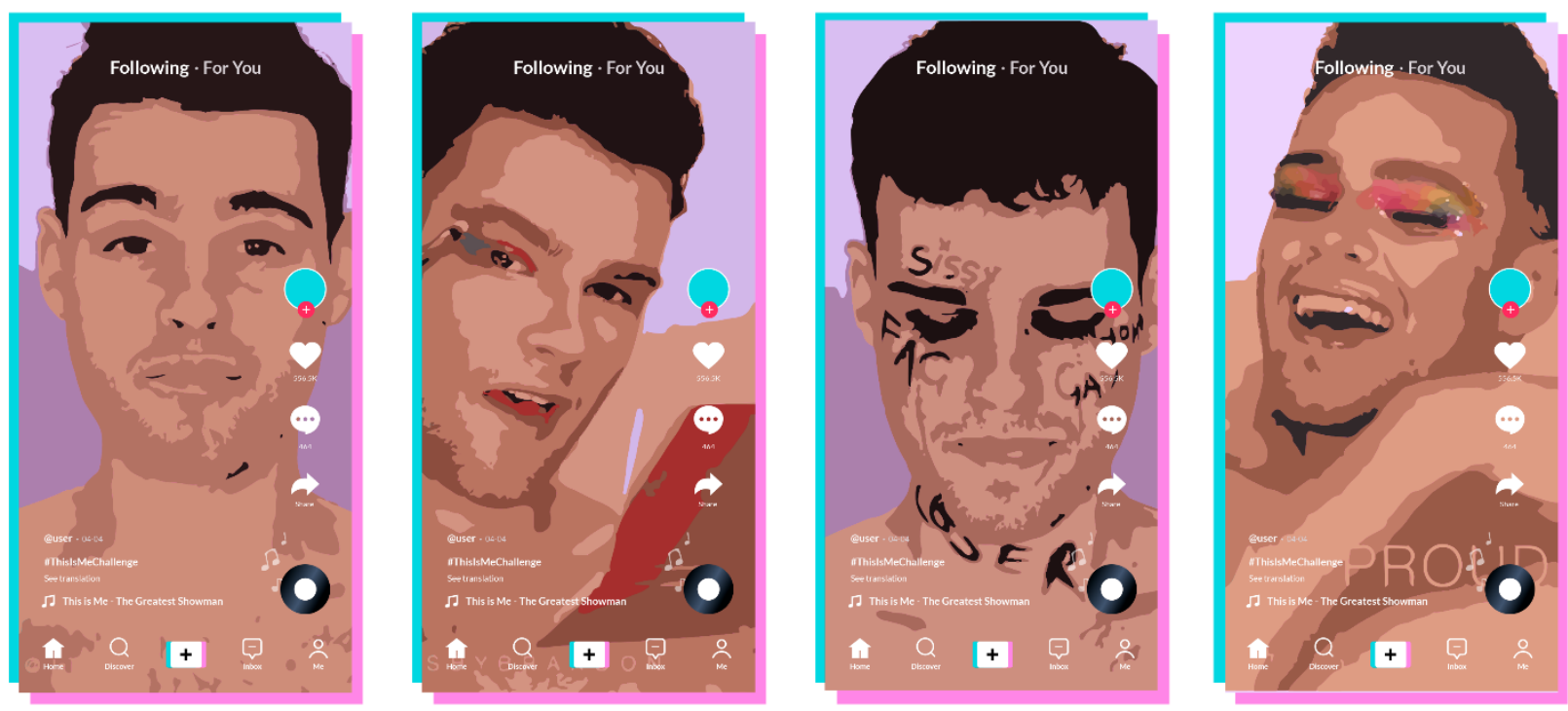

Figure 3. Main stages of \#ThislsMeChallenge. From left to right: TikTokers appearing in front of the camera in a close-up shot; singing the song as they make-up through several transitions; covering their face with negative words; appearing in colorful make-up, smiling, and singing happily.

$(n=9)$, colonialism $(n=2)$, homophobia $(n=30)$, ableism $(n=5)$, religion discrimination $(n=1)$, gender identity ( $n=27)$, bullying $(n=26)$, culture $(n=2)$, other $(n=9)$. We observed cases where the same post could be classified into more than one category, e.g., a transgender TikToker could be targeted in homophobic and gender identity issues.

\subsubsection{Racism}

Racism is understood as an aggravation of the rational sense of an ethnic group that usually motivates the discrimination of individuals, which is usually extended to groups. In this sense, we are referring to the anthropological-philosophical perspective based on racial supremacy, which is scientifically fallacious and socially iniquitous, unjust, and dangerous (Urquidez, 2021). The most significant and generalizable correla- tion was between racism and reflexivity: $r s(100)=.921$, $p<.001, r^{2}=.95,1-\beta=.1$. The TikTokers identified, besides negative words, their foreign status in a different country and in many cases its consequences (Figure 4). Many of the racism-related posts referenced accusations of theft and unjustified hatred towards physical aspects such as skin color. In fact, there were examples involving the \#BlackLivesMatter message-which is a decentralized international movement to ensure justice and equality for black people since the death of the African American Trayvon Martin (Gatwiri et al., 2021).

Another statistically significant relationship was between racism and capability, $r s(100)=.836, p<.001$, $r^{2}=.92,1-\beta=.1$, through which self-described TikTokers highlighted their aptitude to adapt to foreign environments in spite of adversity and discrimination. Subsequently, in order of statistical significance and sample relevance, we highlighted tradition, $r s(100)=.521$,


Figure 4. Illustrated TikTok post about racism and reflexivity. 
$p<.001, r^{2}=.72,1-\beta=.1$; other, $r s(100)=.521, p<.001$, $r^{2}=.72,1-\beta=.1$; equality, $r s(100)=.463, p<.001$, $r^{2}=.68,1-\beta=.99 ;$ pride, $r s(100)=.303, p<.002, r^{2}=.55$, $1-\beta=.99$; and acknowledgement, $r s(100)=.68, p<.001$, $r^{2}=.82,1-\beta=.1$.

\subsubsection{Colonialism}

Colonialism is defined as a social and economic system whereby a foreign state dominates and operates a territory, usually by military, political, economic, and social pressure, appropriating the land, resources, and culture of a community (Mouton \& Burns, 2021). This variable showed a significant and meaningful relationship between colonialism and tradition, $r s(100)=.99$, $p<.001, r^{2}=.99,1-\beta=.1$, and colonialism and other, $r s(100)=.99, p<.001, r^{2}=.99,1-\beta=.1$, in which TikTokers discussed the traditional aspects appropriated from their ethnicity or territory (Figure 5). Compared to studies that have analyzed data colonialism (which consists of the commodification of the minority groups' information for capitalist purposes; Couldry \& Mejias, 2019), we observed traditional protests about the cultural, territorial, and historical discrimination experi- enced by native communities.

It was followed by the correlations less statistically significant such as capability, $r s(100)=.623, p<.001$, $r^{2}=.78,1-\beta=.1$; reflexivity, $r s(100)=.565, p<.001$, $r^{2}=.75,1-\beta=.1$; acknowledgement, $r s(100)=.354$, $p<.001, r^{2}=.59,1-\beta=.99 ;$ and equality, $r s(100)=.241$, $p<.001, r^{2}=.49,1-\beta=.99$. In this case, we did not find a significant relationship between colonialism and pride, as the results were not consistent in terms of $p<.05$.

\subsubsection{Homophobia}

Homophobia refers to the hostility towards homosexuality of both women and men, though it usually extends to people of sexual diversity (bisexual, transsexual, and transgender individuals), which in the latter two cases is referred to as transphobia. The term refers to the irrational rejection of these groups, frequently entailing violence and discrimination (Sell \& Krims, 2021). The relationship between homophobia and equality was statistically significant, $r s(100)=.896, p<.001, r^{2}=.94,1-\beta=.1$, as many of the TikTokers identified in this category urged to end the hate towards them (Figure 6). This idea is supported by the great presence of LGBTQ+ collectives on
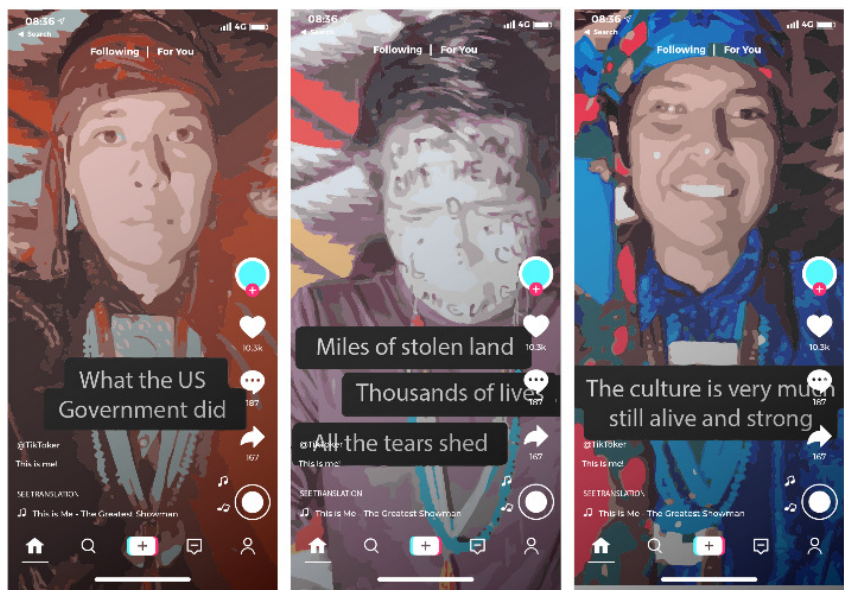

Figure 5. Illustrated TikTok post about colonialism and tradition.
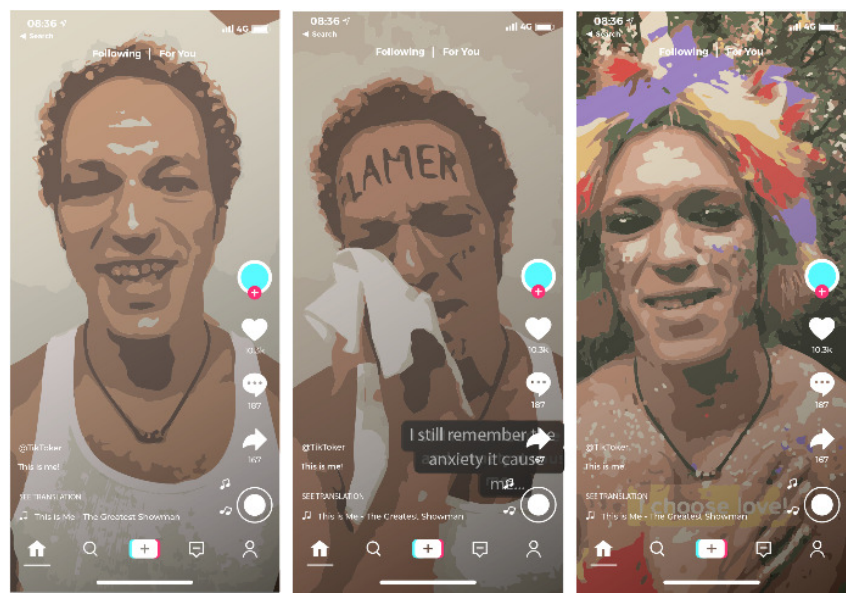

Figure 6. Illustrated TikTok post about homophobia and equality. 
social media, where there is a praxis for creating, sharing, and caring for each other in community (Jenzen \& Karl, 2014).

Also noteworthy, ranked by significance, were the relationships related to acknowledgement, $r s(100)=.760, p<.001, r^{2}=.87,1-\beta=.1$; pride, $r s(100)=.587, p<.002, r^{2}=.76,1-\beta=.1$; reflexivity, $r s(100)=.476, p<.001, r^{2}=.68,1-\beta=.99$; capability, $r s(100)=.432, p<.001, r^{2}=.65,1-\beta=.99$; tradition, $r s(100)=.269, p<.001, r^{2}=.51,1-\beta=.99 ;$ and other, $r s(100)=.269, p<.001, r^{2}=.51,1-\beta=.99$.

\subsubsection{Ableism}

Ableism is a form of discrimination or social prejudice against people with disabilities (Cooper-Stoll \& Egner, 2021), who are those that have developed a physical, intellectual, or sensory deficiency (lezzoni et al., 2021). For this variable we found a significant and relevant relationship between ableism and capability, $r s(100)=.99, p$ $<.001, r^{2}=.99,1-\beta=.1$, where TikTokers showed their professional success despite the difficulties (Figure 7).

Next, there were significant and statistical relationships with reflexivity, $r s(100)=.908, p<.001, r^{2}=.95$, $1-\beta=.1$; other, $r s(100)=.623, p<.001, r^{2}=.78,1-\beta=.1$; tradition, $r s(100)=.623, p<.001, r^{2}=.78,1-\beta=.1$; acknowledgement, $r s(100)=.569, p<.001, r^{2}=.75$, $1-\beta=.1$; equality, $r s(100)=.387, p<.001, r^{2}=.62$, $1-\beta=.99$; and pride categories, $r s(100)=.254, p<.002$, $r^{2}=.50,1-\beta=.99$.

\subsubsection{Religion Discrimination}

Religion is defined as a cultural system characterized by behaviors and practices, worldviews, ethics, texts, and other human history events that constitute group experiences, meanings, convictions, and beliefs based on a selftranscending dialectic (Paloutzian \& Park, 2021). For this group we identified a significant relationship between religion and tradition, $r s(100)=.704, p<.001, r^{2}=.83$, $1-\beta=.1$. In this case, the TikTokers showed their beliefs as part of themselves mostly on a spiritual perspective (Figure 8).

Statistically less significant relationships were found in terms of other, $r s(100)=.701, p<.001, r^{2}=.83$, $1-\beta=.1$; capability, $r s(100)=.438, p<.001, r^{2}=.66$, $1-\beta=.99$; reflexivity, $r s(100)=.398, p<.001, r^{2}=.63$, $1-\beta=.99 ;$ and acknowledgement, $r s(100)=.249, p<.001$,


Figure 7. Illustrated TikTok post about ableism and capability.


Figure 8. Illustrated TikTok post about religion and tradition. 
$r^{2}=.49,1-\beta=.99$. We found no relationship with equality and pride in the absence of the statistical assumption, $p<.05$.

\subsubsection{Gender Identity}

Using a social sciences approach, we understand gender identity as a concept that depicts human identity groups based on socially defined roles, behaviors, activities, and physical-psychological attributes (female, male, non-binary, and gender fluid; Kime-Scott, 2021). The most significant correlation was between gender and equality: $r s(100)=.975, p<.001, r^{2}=.98,1-\beta=.1$. Similar to the homophobia variable, the TikTokers claimed equality because of their gender (Figure 9). In fact, the second relationship emphasized was between gender and pride: $r s(100)=.672, p<.002, r^{2}=.81$, $1-\beta=.1$. Addressing the inclusion of an unsatisfactory democratic gendered citizenship approach to expressing digital self-identity debates (Ortega-Sánchez et al., 2021), in this study we found a positive and proactive counternarrative. The TikTokers who were classified in this group explained their identity, or else how they found themselves, i.e., their experience in identifying their gender in a healthy and comfortable manner.
The rest of the variables that were significantly correlated were related to acknowledgement, $r s(100)=.663$, $p<.001, r^{2}=.81,1-\beta=.1$; reflexivity, $r s(100)=.415$, $p<.001, r^{2}=.64,1-\beta=.99$; capability, $r s(100)=.377$, $p<.001, r^{2}=.61,1-\beta=.99 ;$ tradition, $r s(100)=.235$, $p<.001, r^{2}=.48,1-\beta=.99 ;$ and other, $r s(100)=.235$, $p<.001, r^{2}=.48,1-\beta=.99$.

\subsubsection{Bullying}

Bullying is any form of psychological, verbal, or physical mistreatment directed towards a person repeatedly over a defined period of time in physical or digital space (cyberbullying). This kind of violence is characterized by targeting a person through intimidation, abuse of power and physical aggression, among others (Ansary, 2020). Bullying was significantly correlated with equality, $r s(100)=.99, p<.001, r^{2}=.99,1-\beta=.1$, followed by acknowledgment, $r s(100)=.681, p<.001, r^{2}=.82$, $1-\beta=.1$, and pride, $r s(100)=.655, p<.002, r^{2}=.80$, $1-\beta=.1$. In these cases, the TikTokers highlighted the physical or psychological abuse perpetrated against them (Figure 10).

The other relationships, which were less significant, were related to reflexivity, $r s(100)=.426, p<.001$,


Figure 9. Illustrated TikTok post about gender and equality.
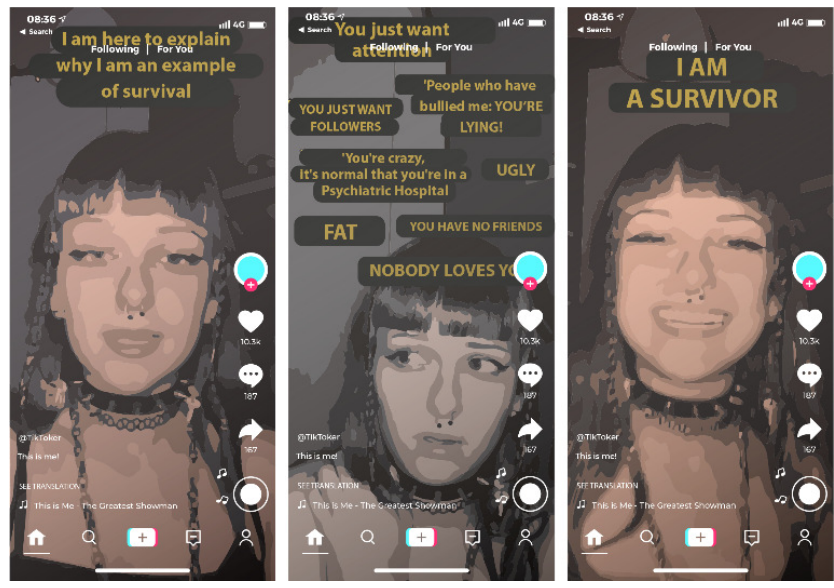

Figure 10. Illustrated TikTok post about bullying and equality. 
$r^{2}=.65,1-\beta=.99 ;$ capability, $r s(100)=.387, p<.001$,

$r^{2}=.62,1-\beta=.99 ;$ tradition, $r s(100)=.241, p<.001$,

$r^{2}=.49,1-\beta=.97$; and other, $r s(100)=.241, p<.001$, $r^{2}=.49,1-\beta=.97$.

\subsubsection{Culture}

Culture is a polysemous term extensively discussed by anthropologists, which is mainly grounded in the appreciation of fine arts and humanities, and in the knowledge, beliefs, and behaviors of a social group (Fox \& King, 2020). We found a statistically significant relationship between culture and tradition, $r s(100)=.99, p<.001, r^{2}=.99$, $1-\beta=.1$, and culture and other, $r s(100)=.99, p<.001$, $r^{2}=.99,1-\beta=.1$. In the latter case we found a cosplayer, who is a person belonging to a Japanese culture phenomenon associated with dressing up as fandom characters, in which the TikToker showed her skills (Figure 11).

Relatively less significant were the relationships with capability, $r s(100)=.623, p<.001, r^{2}=.78,1-\beta=.1$; reflexivity, $r s(100)=.565, p<.001, r^{2}=.75,1-\beta=.1$; acknowledgement, $r s(100)=.354, p<.001, r^{2}=.59$, $1-\beta=.99 ;$ and equality, $r s(100)=.241, p<.001, r^{2}=.49$, $1-\beta=.99$. The pride variable was excluded for not complying to the assumption of $p<.05$.

\subsubsection{Other}

Finally, we included those issues not considered previously, listing particular cases related to acknowledgment, $r s(100)=.99, p<.001, r^{2}=.99,1-\beta=.1$. We identified hate towards influencers for their popularity on TikTok (Figure 12), as well as skin-related and other diseases (alopecia or vitiligo), in which they proudly showed their identity and/or problem.

Less remarkable were the relations with equality, $r s(100)=.681, p<.001, r^{2}=.82,1-\beta=.1$; reflexivity, $r s(100)=.626, p<.001, r^{2}=.79,1-\beta=.1$; capability, $r s(100)=.569, p<.001, r^{2}=.75,1-\beta=.1$; pride, $r s(100)=.446, p<.002, r^{2}=.66,1-\beta=.99 ;$ tradition, $r s(100)=.354, p<.001, r^{2}=.59,1-\beta=.99$; and other categories, $r s(100)=.354, p<.001, r^{2}=.59,1-\beta=.99$.

\subsection{Supporting Marginalized Groups Through Polarity and Subjectivity}

We analyzed the polarity and subjectivity of 8,877 comments to find out whether users viewing the 100 posts turned their sympathy toward empathy (Chanan, 2011; Olinick, 2014). First, we averaged both cases. Taking the SA criteria, by which values from 0 to 1 indicate positive
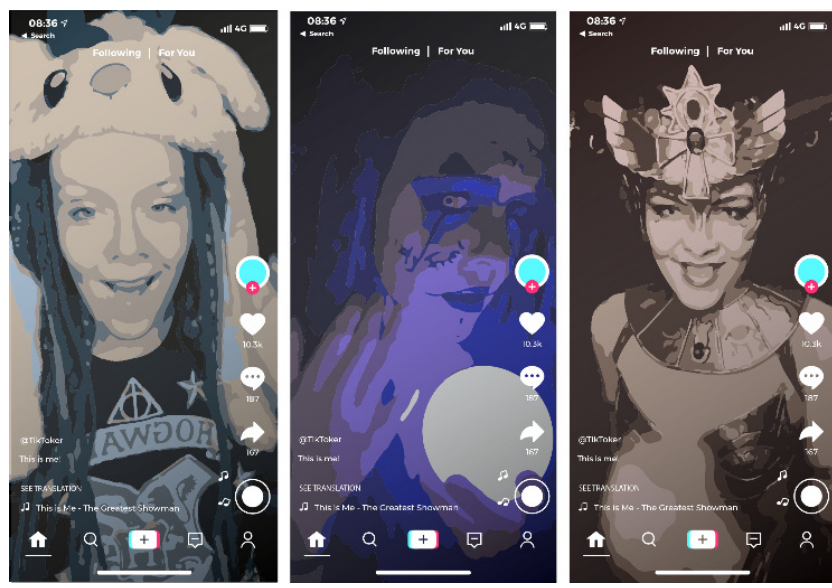

Figure 11. Illustrated TikTok post about culture and other (cosplay).
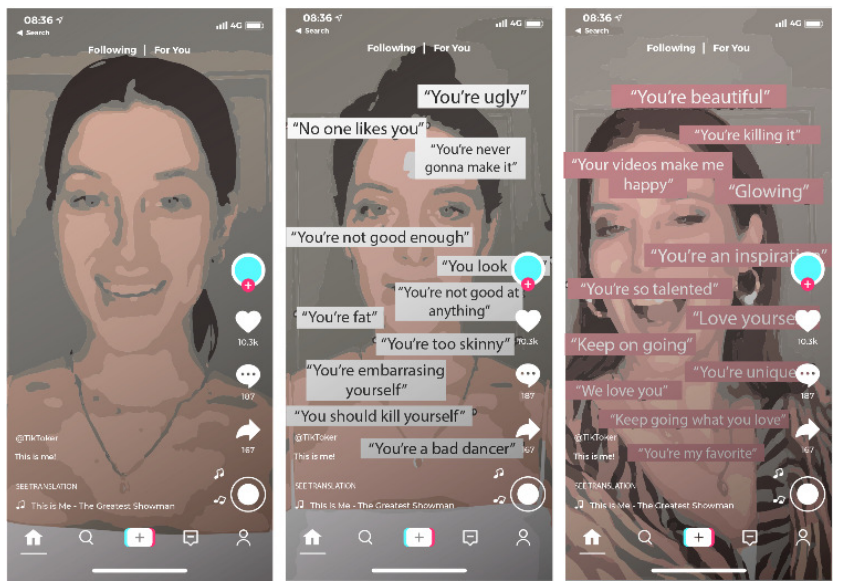

Figure 12. Illustrated TikTok post about other and acknowledgment. 
content, and from 0 to -1 for negative content, we found a mean of .528 for polarity. Likewise, we found a mean of .788 for subjectivity. This means that the extensive community posted primarily positive insights with high subjective value. Beyond these data, we choose the most positive comments to understand if sympathy (positivity) reflected support for the identity/injustice shared by the TikTokers (Figure 13).

In contrast to the negative words introduced by the TikTokers, in the comments we found a large number of positive adjectives and emoji characterized, for example, by the LGBTIQ+ flag, and supportive speech (up to 784 comments reported 1 polarity point, i.e., maximum positivity). We found a wide variety of comments that emphasized the beauty of the TikTokers, e.g., "beautiful," "awesome," "amazing," "spectacular :-)," "gorgeous," "perfect " among others. We also found terms that referred to the empowerment of these marginalized groups with messages such as "powerful and strong message," "keep fighting for your dreams," or "it's empowering 20 ." On the other hand, we found expressions that praised the content created by TikTokers: "your transitions are amazing 10060 , "look at that picture quality," "those transitions are cleaner than my face (2)," "super talented $\bigcirc$," and "you are a crack and you deserve to be duetted." In most cases we observed that comments introduced multiple emoji with attitudinal meaning that differed from the linguistic prosodic pattern, making evident other emotions (Logi \& Zappavigna, 2021). For the cases previously reported, we found facial expressions that consumer science scholars Jaeger and Ares (2017) classified as (a) $\odot=$ happy, satisfied; (b) $\Theta$ = excited, happy; and (c) (20) = surprised, shocked. As a result, we observed a high degree of subjectivity in the comments, as well as a clear positive attitude, showing the community's support for the empowerment of these groups. These data demonstrated growing activist support for marginalized groups through expressions of free and inclusive self-choice (Givropoulou \& Tseliou, 2021).

\section{Conclusions}

In this research we explored the role of music for cybercitizen empowerment within TikTok and the \#ThisIsMeChallenge trend. This is a phenomenon based on the transmedia music concept (Vizcaíno-Verdú et al., 2021), whereby fictional songs serve as a driver for appropriating, creating, and sharing meaningful content. Departing from the "This Is Me" song theme and drawing explicit references to the self-improvement and selfesteem of traditionally marginalized groups, we focused on anthropological and sociological aspects related to participatory culture. This approach revealed how identities are shared through social movements that add cultural value using transmedia music (Vizcaíno-Verdú \& Abidin, 2021; Vizcaíno-Verdú et al., 2021). Despite the animosity and the dark side of social media emphasized by Kilvington (2021) and Weimann and Masri (2020), in this analysis we have found the opportunities involving music-making interactions for citizen democratization (Chambers, 2021).

Our results reflect the contrasting nature of stories told by TikTokers, which are turned into a motif for reflexivity, equality, capability, acknowledgement, and pride, with the unconditional encouragement of users commenting the posts. If the personality shared through the pre-existing song (Anderson et al., 2020) already makes

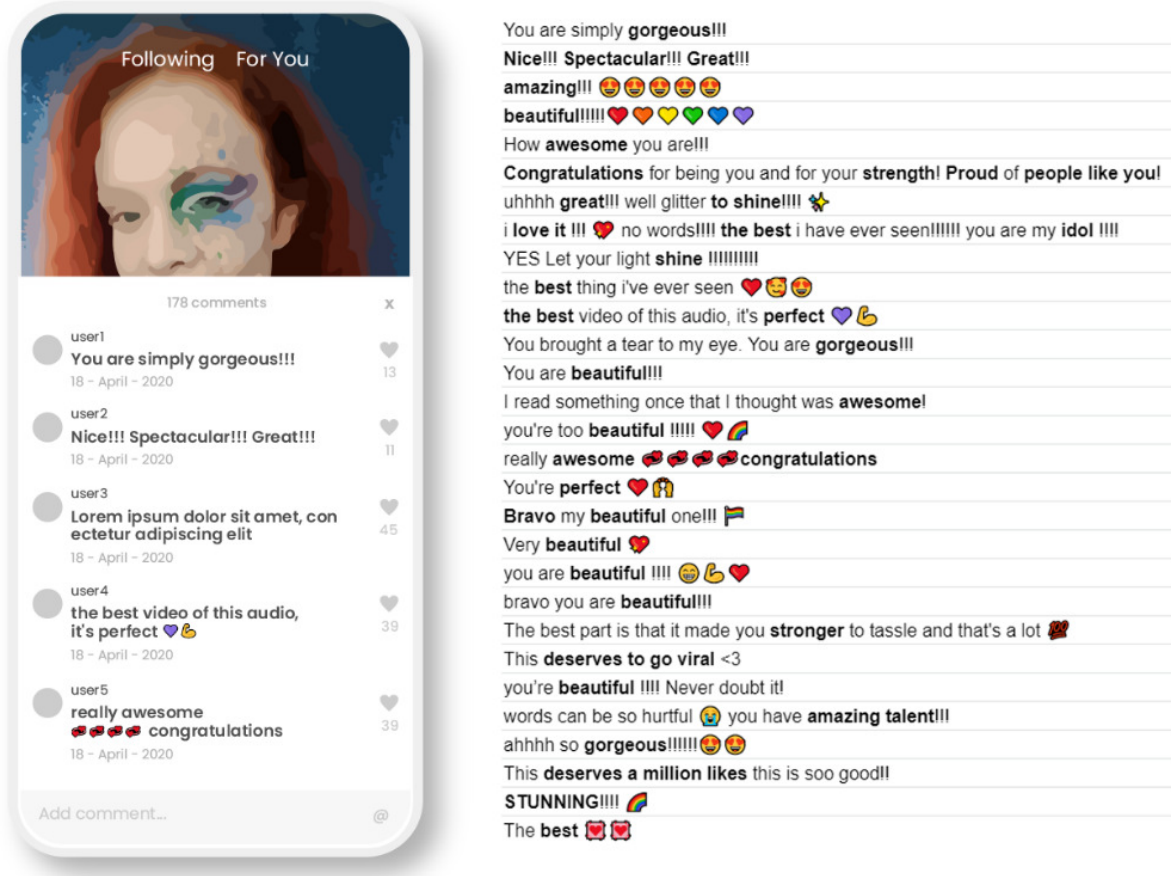

Figure 13. Top positive comments on \#ThislsMeChallenge. 
us aware of how strong and capable the TikTokers are, even more so the thousands of comments contributing to the support of the individual or collective identity (Asún \& Zúñiga, 2013). Albeit the interaction was asynchronous and there was no direct relationship between the TikToker and user (because the amount of comments may not necessarily be associated with true followers in view of the platform's varied content in the "For You Page" feed), the volunteer comments showed the result of what Maiorano et al. (2021) described as empowerment. Precisely, we relied on aspects related to changing preconceived prejudices about the human condition. In other words, the TikTokers shared fears, anxieties, and negative experiences that were supported by messages that ensured diversity, equality, affection and, ultimately, empathy (Chanan, 2011). As such, we could consider that these empowering practices, in which the assumedly normal and real content prevails, disrupts the "aggressive architecture" of the internet (Harvey, 2019). In this sense, we might also suspect that such marginalized groups occupy a high priority for TikTok, especially in the prevention of social discrimination.

In this context, we are faced with a transmediaempowered video activism approach in which music is motivating individuals and collectives to learn about diversity in cultures and societies. This means that users have initiated a phenomenon that consists in the appropriation of an audio meme (Zulli \& Zulli, 2020) inspired by the soundtrack of an original fiction text to express their emotions through a film's story about self-esteem (Vizcaíno-Verdú et al., 2021). Considering the injustices, individual coping, and community support observed, it seems that we are moving towards a transmedia music phenomenon that motivates the empowerment of traditionally marginalized groups (Spears \& Postmes, 2015). While this music challenge became a trend with extensive views and engagement, the \#ThislsMeChallenge phenomenon emerging from a film's song lyrics represented a small sample of a broader international social movement related to the decolonization of the global south, LGBTQ+, \#MeToo, or \#BlackLivesMatter protests.

This study's major limitations are in terms of the sample, statistical power, and SA. Although the statisticalmethodological proposal provided insights into a phenomenon grounded in the era of ephemeral content and memes, we found that the limited number of TikTok videos was not statistically significant for all the marginalized groups proposed. In fact, we observed that some of the previously introduced social discriminations (Velasquez \& Montgomery, 2020) were not found in the sample. This issue was mainly due to the limited posts created for \#ThislsMeChallenge. In addition, we noted that the SA approach was created by predesigned algorithms that did not provide a semiotic discourse analysis of aspects such as emojis (Logi \& Zappavigna, 2021), which produced biases between polarity-subjectivity and reality. Accordingly, we suggest that future research approaches music empowerment on social media using the proposed codebook, as well as diving deeper into the aspects that make music a transmedia storytelling tool for platforms such as TikTok.

\section{Acknowledgments}

We thank the anonymous referees for their useful suggestions, which improved the quality of the article. We would also like to thank Daniela Jaramillo-Dent for her valuable and thoughtful comments in reviewing the final version.

\section{Funding}

This work was supported by Alfamed (Euro-American Research Network), under Grant $\mathrm{R}+\mathrm{D}+\mathrm{I}$ Project (2019-2021), entitled Youtubers and Intagrammers: Media competition in emerging prosumers, with code RTI2018-093303-B-I00, financed by the Spanish Ministry of Science, Innovation and Universities and the European Regional Development Fund (ERDF), and the R+D-i Project (2020-2022), entitled Instagrammers and youtubers for the transmedia empowerment of Andalusian citizens. The media competence of instatubers, with code P18-RT-756, financed by the Andalusian Regional Government in the 2018 call (Andalusian Research, Development and Innovation Plan, 2020) and the European Regional Development Fund (ERDF).

\section{Conflict of Interests}

The authors declare no conflict of interests.

\section{References}

Abidin, C. (2016). Visibility labour: Engaging with influencers' fashion brands and \#OOTD advertorial campaigns on Instagram. Media International Australia, 161(1), 86-100. https://doi.org/10.1177/ $1329878 \times 16665177$

Abidin, C. (2021). Mapping internet celebrity on TikTok: Exploring attention economies and visibility labours. Cultural Sciences, 12(1), 77-103. https://doi.org/ 10.5334/csci.140

Anderson, I., Gil, S., Gibson, C., Wolf, S., Shapiro, W., Semerci, O., \& Greenberg, D. M. (2020). "Just the way you are": Linking music listening on Spotify and personality. Social Psychological and Personality Science, 12(4), 561-572. https://doi.org/10.1177/ 1948550620923228

Anderson, K. E. (2020). Getting acquainted with social networks and apps: It is time to talk about TikTok. Library Hi Tech News, 37(4), 7-12. https://doi.org/ 10.1108/LHTN-01-2020-0001

Ansary, N. S. (2020). Cyberbullying: Concepts, theories, and correlates informing evidence-based best practices for prevention. Aggression and Violent Behavior, 50, Article 1011343. https://prohic.nl/ 
wp-content/uploads/2020/11/2020-04-22-

CyberbullyingMeta.2020.pdf

Askanius, T. (2013). Online video activism and political mash-up genres. Journalism, Media and Cultural Studies, 4, 1-20. https://doi.org/10.18573/ j.2013.10257

Asún, R., \& Zúñiga, C. (2013). Why participate? Explaining regionalist social protest from two psychosocial models. Psicoperspectivas, Individuo y Sociedad, 12(2), 38-50. https://doi.org/10.5027/ PSICOPERSPECTIVAS-VOL12-ISSUE2-FULLTEXT-260

Birjali, M., Kasri, M., \& Beni-Hssane, A. (2021). A comprehensive survey on sentiment analysis: Approaches, challenges and trends. KnowledgeBased Systems, 227, Article 107134. https://doi.org/ 10.1016/j.knosys.2021.107134

Bock, A., Isermann, H., \& Knieper, T. (2011). Quantitative content analysis of the visual. In E. Margolis \& L. Pauwels (Eds.), The SAGE handbook of visual research methods (pp. 265-282). SAGE. https://doi. org/10.4135/9781446268278.n14

Born, G., \& Tilley, C. (2011). Music and the materialization of identities. Journal of Material Culture, 16(4), 376-388. https://doi.org/10.1177/ 1359183511424196

boyd, d. (2013). It's complicated: The social lives of networked teens. Yale University Press.

Chambers, P. (2021). Producing the self: Digitisation, music-making and subjectivity. Journal of Sociology. Advance online publication. https://doi.org/ $10.1177 / 14407833211009317$

Chanan, M. (2011). Video, activism, and the art of small media. Transnational Cinemas, 2(2), 217-226. https://doi.org/10.1386/trac.2.2.217_7

Civila, S., Romero-Rodríguez, L. M., \& Civila, A. (2020). The demonization of Islam through social media: A case study of \#Stoplslam in Instagram. Publications, 8(4), Article 52. https://doi.org/10.3390/ publications 8040052

Cooper-Stoll, L., \& Egner, J. (2021). We must do better: Ableism and fatphobia in sociology. Sociology Compass, 15(4), Article e12869. https://doi.org/10.1111/ soc4.12869

Couldry, N., \& Mejias, U. A. (2019). Data colonialism: Rethinking big data's relation to the contemporary subject. Television \& New Media, 20(4), 336-349. https://doi.org/10.1177/1527476418796632

Craig, S. L., Eaton, A. D., McInroy, B., Leung, V. W. Y., \& Krishnan, S. (2021). Can social media participation enhance LGBTQ+ youth well-being? Development of the social media benefits scales. Social Media + Society. Advance online publication. https://doi.org/ $10.1177 / 2056305121988931$

Fang, J., Wang, Z., \& Hao, B. (2019). Analysis of "anesthesia" mechanism in mobile short video applications. In T. Strielkowski \& J. Cheng (Eds.), Advances in social science, education and humanities research (pp. 348-351). Atlantis Press. https://doi.org/10.2991/ ismss-19.2019.75

Fleiss, J. L., Levin, B., \& Paik, M. C. (2003). Statistical methods for rates and proportions. Wiley. https://doi.org/ 10.1002/0471445428

Fox, R. G., \& King, B. J. (2020). Anthropology beyond culture. Body, age and identity. Routledge. https:// doi.org/10.4324/9781003084631

García-Galera, M. C., Fernández-Muñoz, C., \& PortoPedrosa, L. (2017). Youth empowerment through social networks. Creating participative digital citizenship. Communication \& Society, 30(3), 129-140. https://doi.org/10.15581/003.30.3.129-140

García-Moreno, H. (2020). La traducción musical: El caso de The Greatest Showman [Music translation: The case of The Greatest Showman]. Universitat Pompeu Fabra.

Gatwiri, K., Rotumah, D., \& Rix, E. (2021). BlackLivesMatter in healthcare: Racism and implications for health inequity among aboriginal and Torres strait islander peoples in Australia. International Journal of Environmental Research and Public Health, 18(9), Article 4399. https://doi.org/10.3390/ijerph18094399

George, J., George, T., \& Moquin, R. (2021). I can't breathe: How digital video becomes an emancipatory technology. In Proceedings of the 54th Hawaii international conference on system sciences (pp. 6369-6378). HICSS. https://doi.org/10.24251/HICSS. 2021.768

Givropoulou, D., \& Tseliou, E. (2021). Developing reflexivity through group processes in psychotherapy training: An interpretative phenomenological analysis of systemic family therapy trainees' experience. Family Process, 60(2), 346-360. https://doi.org/10.1111/ famp. 12600

Harb, B., \& Sidani, D. (2020). Smart technologies challenges and issues in social inclusion-Case of disabled youth in a developing country. Journal of Asia Business Studies. Advance online publication. https://doi.org/10.1108/JABS-10-2020-0389

Harvey, A. (2019). Tits or GTFO: The aggressive architecture of the internet. FLOW. https://bit.ly/32ECy5X

lezzoni, L. I., Rao, S. R., Ressalam, J., Bolcic-Jankovic, D., Agaronnik, K. D., Lagu, T., \& Campbell, E. G. (2021). Physicians' perceptions of people with disability and their health care. Health Affairs, 40(2), 1-15. https:// doi.org/10.1377/hlthaff.2020.01452

Jaeger, S. R., \& Ares, G. (2017). Dominant meanings of facial emoji: Insights from Chinese consumers and comparison with meanings from internet resources. Food Quality and Preference, 62, 275-283. https:// doi.org/10.1016/j.foodqual.2017.04.009

Jaramillo-Dent, D., Contreras-Pulido, P., \& PérezRodríguez, A. (2021). Right-wing immigration narratives in Spain: A study of persuasion on Instagram stories. European Journal of Communication. Advance online publication. https://doi.org/10.1177/026732 31211012157

Jenzen, O., \& Karl, I. (2014). Make, share, care: Social 
media and LGBTQ youth engagement. Ada, A Journal of Gender, New Media, and Technology, 5. Advance online publication. https://doi.org/10.7264/ N39P2ZX3

Kennedy, M. (2020). If the rise of the TikTok dance and egirl aesthetic has taught us anything, it's that teenage girls rule the internet right now: TikTok celebrity, girls and the Coronavirus crisis. European Journal of Cultural Studies, 23(6), 1069-1076. https://doi.org/ $10.1177 / 1367549420945341$

Kilvington, D. (2021). The virtual stages of hate: Using Goffman's work to conceptualize the motivations for online hate. Media, Culture \& Society, 43(2), 256-272. https://doi.org/10.1177/016344372097 2318

Kime-Scott, B. (2021). Introduction to the gender of modernism. In S. Latham \& G. Rogers (Eds.), The new modernist studies reader. An anthology of essential criticism (pp. 28-40). Bloomsbury Publishing.

Krippendorff, K. (2004). Content analysis: An introduction to its methodology. SAGE.

Kumari, M. (2020). Social media and women empowerment. International Journal of Scientific \& Technology Research, 9(3), 626-629. https://bit.ly/2ULUY05

Light, B., Burgess, J., \& Duguay, S. (2016). The walkthrough method: An approach to the study of apps. New Media \& Society, 20(3), 881-900. https://doi. org/10.1177/1461444816675438

Literat, I., Abdelbagi, A., Law, N. Y. L., Cheung, M. Y. Y., \& Tang, R. (2021). Research note: Likes, sarcasm and politics: Youth responses to a platform-initiated media literacy campaign on social media. Harvard Kennedy School Misinformation Review, 2(3), 1-10. https://doi.org/10.37016/mr-2020-67

Literat, I., \& Kligler-Vilenchik, N. (2021). How popular culture prompts youth collective political expression and cross-cutting political talk on social media: A cross-platform analysis. Social Media + Society, 7(2) 1-14. https://doi.org/10.1177/20563051211008821

Logi, L., \& Zappavigna, M. (2021). A social semiotic perspective on emoji: How emoji and language interact to make meaning in digital messages. New Media \& Society. Advance online publication. https://doi.org/ 10.1177/14614448211032965

Maiorano, D., Shrimankar, D., Thapar-Björkert, S., \& Blomkvist, H. (2021). Measuring empowerment: Choices, values and norms. World Development, 138, Article 105220. https://doi.org/10.1016/j.worlddev. 2020.105220

Mancini, T., \& Imperato, C. (2020). Can social networks make us more sensitive to social discrimination? E-contact, identity processes and perception of online sexual discrimination in a sample of Facebook users. Social Sciences, 9(4), 47. https://doi.org/ 10.3390/socsci9040047

Medhat, W., Hassan, A., \& Korashy, H. (2014). Sentiment analysis algorithms and applications: A survey. Ain Shams Engineering Journal, 5, 1093-1113. https:// doi.org/10.1016/j.asej.2014.04.011

Meneses-Rocha, M. E., \& Castillo-González, M. C. (2016). \#TodossomosAyotzinapa. Storytelling, identities, representations and reflexivity in dispute. Cultural Studies Journal of Universitat Jaume I, 26, 37-56. https:// doi.org/10.6035/CLR.2016.16.3

Meyer, M. (2019). The impact of social media on non-monosexuals' responses to discrimination: $A$ co-cultural approach. Social Media + Society, 5(1), 1-15. https://doi.org/10.1177/2056305119826120

Mouton, M., \& Burns, R. (2021). (Digital) neo-colonialism in the smart city. Regional Studies, 55(12), 1890-1901. https://doi.org/10.1080/00343404. 2021.1915974

Neuendorf, K. A. (2016). The content analysis guidebook. SAGE. https://doi.org/10.4135/9781071802878

Olinick, M. D. (2014). A critique of empathy and sympathy. In J. D. Lichtenberg, M. Bornstein, \& D. Silver (Eds.), Empathy (pp. 137-166). Routledge.

Ortega-Sánchez, D., Blanch, J. P., Quintana, J. I., Cal, E. S., \& De-la-Fuente-Anuncibay, R. (2021). Hate speech, emotions, and gender identities: A study of social narratives on Twitter with trainee teachers. International Journal of Environmental Research and Public Health, 18, 1-18. https://doi.org/10.3390/ ijerph18084055

Paloutzian, R. F., \& Park, C. L. (2021). The psychology of religion and spirituality: How big the tent? Psychology of Religion and Spirituality, 13(1), 3-13. https:// doi.org/10.1037/rel0000218

Parson, L. (2019). Considering positionality: The ethics of conducting research with marginalized groups. In L. Atkins, \& V. Duckworth (Eds.), Research methods for social justice and equity in education (pp. 15-32). Springer. https://doi.org/10.1007/9783-030-05900-2_2

Place, K. R. (2021). "People are more than just a statistic": Ethical, care-based engagement of marginalized publics on social media. Journal of Media Ethics, 36(3), 141-153. https://doi.org/10.1080/23736992. 2021.1937175

Poria, S., Cambria, E., Bajpai, R., \& Hussain, A. (2017). A review of affective computing: From unimodal analysis to multimodal fusion. Information Fusion, 37, 98-125. https://doi.org/10.1016/j.inffus.2017. 02.003

Sachs, J., Wise, R., \& Karell, D. (2021). The TikTok self: Music, signaling, and identity on social media. SocaArXiv. https://doi.org/10.31235/osf.io/2rx46

Sell, R. L., \& Krims, E. I. (2021). Structural transphobia, homophobia, and biphobia in public health practice: The example of COVID-19 surveillance. American Journal of Public Health, 111(9), 1620-1626. https://doi.org/10.2105/AJPH.2021.306277

Smith, M. A., Williamson, L. D., \& Bigman, C. A. (2020). Can social media news encourage activism? The impact of discrimination news frames on college students' activism intentions. Social Media + 
Society, 6(2), 1-10. https://doi.org/10.1177/20563 05120921366

Spears, R., \& Postmes, T. (2015). Group identity, social influence, and collective action online. In S. S. Sundar (Ed.), The handbook of the psychology of communication technology (pp. 23-46). https://doi.org/ 10.1002/9781118426456.ch2

Urquidez, A. G. (2021). Reply to my critics: (Re-)Defining racism: A philosophical analysis. Ethic Theory Moral Practice, 24, 679-698. https://doi.org/ 10.1007/s10677-021-10207-2

Velasquez, A., \& Montgomery, G. (2020). Social media expression as a collective strategy: How perceptions of discrimination and group status shape US Latinos' online discussions of immigration. Social Media + Society, 6(1), 1-14. https://doi.org/10.1177/ 2056305120914009

Vizcaíno-Verdú, A., \& Abidin, C. (2021, October 13-16). Cross-cultural storytelling approaches in TikTok's music challenges [Paper presentation]. The 22nd Annual Conference of the Association of Internet Researchers. https://doi.org/10.5210/spir.v2021i0. 12260

Vizcaíno-Verdú, A., Aguaded, I., \& Contreras-Pulido, P. (2021). Understanding transmedia music on YouTube through Disney storytelling. Sustainability, 13(7), Article 3667. https://doi.org/10.3390/su13073667
Wahl-Jorgensen, K. (2020). An emotional turn in journalism studies? Digital Journalism, 8(2), 175-194. https://doi.org/10.1080/21670811.2019.1697626

Waugh, M. (2017). My laptop is an extension of my memory and self: Post-Internet identity, virtual intimacy and digital queering in online popular music. Popular Music, 36(2), 233-252. https://doi.org/10.1017/ S0261143017000083

Weimann, G., \& Masri, N. (2020). Spreading hate on TikTok. Studies in Conflict \& Terrorism. Advance online publication. https://doi.org/10.1080/1057610X. 2020.1780027

Yalcin, O. N., \& DiPaola, S. (2018). A computational model of empathy for interactive agents. Biologically Inspired Cognitive Architectures, 26, 20-25. https:// doi.org/10.1016/j.bica.2018.07.010

Zhang, Z. (2020). Infrastructuralization of Tik Tok: Transformation, power relationships, and platformization of video entertainment in China. Media, Culture \& Society, 43(2), 219-236. https://doi.org/10.1177/ 0163443720939452

Zulli, D., \& Zulli, D. J. (2020). Extending the Internet meme: Conceptualizing technological mimesis and imitation publics on the TikTok platform. New Media \& Society. Advance online publication. https://doi. org/10.1177/1461444820983603

\section{About the Authors}
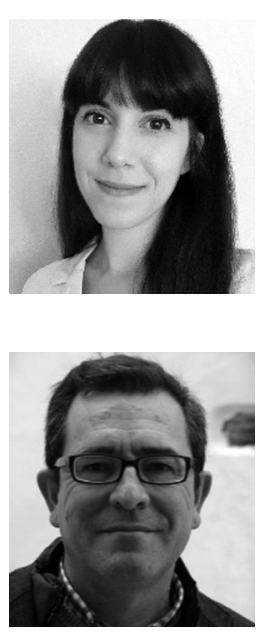

Arantxa Vizcaíno-Verdú is communication predoctoral fellow at the University of Huelva (Spain). She researches transmedia storytelling, fandom, and participatory culture on social media. Her work appears in peer-reviewed journals including Learning, Media \& Technology, Comunicar, Sustainability, Educación XX1, International Journal of Communication, among others. She is associate editor of the media education research journal Comunicar (JCR-Scopus Q1), and key regional leader of the TikTok Cultures Research Network. She is also member of the research group Agora, the Comunicar Group, and the research network Alfamed Junior.

Ignacio Aguaded is full time professor of media education and technologies applied to education at the University of Huelva (Spain). He is the founding president of Comunicar Group, and the editor-in-chief of the media education research journal Comunicar (JCR-Scopus Q1). He has led the research group Agora, and he has conducted various media education projects within the framework of Spanish and international programmes. He has lectured at national and international conferences and universities, publishing several scientific books and articles. 